\title{
Estructura semántica \\ y estrategias infantiles \\ en la solución de problemas \\ verbales de adición
}

Vicente Bermejo
Universidad Complutense de Madrid

\section{Purificación Rodríguez}

Escuela Universitaria del Profesorado de E.G.B. de Segovia

\section{INTRODUCCION}

En las tareas de solución de problemas se han seguido diferentes orientaciones teóricas, de modo que puede hablarse de un enfoque asociacionista (Underwood y Richardson, 1956), conductista (Maltzman, 1955), de los teóricos de la gestalt (Duncker, 1945; Kohler, 1927; Wertheimer, 1945, 1959), y más recientemente de las teorías cognitivas. Pero es sin duda alguna en el marco definido por estas últimas, en donde se han llevado a cabo más investigaciones sobre este tema. Baste recordar, por ejemplo, los estudios encaminados a clarificar, tanto teórica como metodológicamente, cómo se produce la resolución de problemas en el ámbito de la física (Larkin, 1985; McDermot y Larkin, 1978; Novack, 1976), de la geometría (Anderson, Greeno, Kine y Neves, 1981; Bishop, 1983; Greeno, 1977, 1978), de la aritmética (Carpenter y Moser, 1982; De Corte y Verschaffel, 1981; Kinstch y Greeno, 1985; Nesher, 1982; Vergnaud, 1982; Wolters, 1983), etc. Igualmente constituye un dato más del avance relativo de estos estudios la proposición de al menos cinco modelos que desde el enfoque del procesamiento de la información pretenden simular los procesos resolutivos encaminados a la solución de problemas verbales aritméticos (Briars y Larkin, en prensa; De Corte y Verschaffel, 1985; Greeno, Riley y Gelman, 1984; Kintsch y Greeno, 1985; Riley, Greeno y Heller, 1983). Todo ello pone de relieve la atención que desde la década pasada, en todo caso, se está prestando a la adquisición de estas nociones.

Hoy podemos afirmar que los logros alcanzados en este campo no son pocos, incrementándose cada día el acervo de conocimientos referidos a nuestro tema. Carpenter, Hiebert y Moser (1981), Carpenter y Moser $(1982,1983)$ y Riley y otros (1983), entre otros autores, han mostrado que la estructura semántica de los problemas verbales influye tanto en la dificultad relativa de los problemas,

* Dirección de los autores: Departamento de Psicología Evolutiva y Educación. Universidad Complutense de Madrid. Facultad de Psicología. Campus de Somosaguas, 28023 Madrid. 
como en las estrategias usadas por los niños en su resolución. Así, se ha encontrado con que en niños de primero de EGB los problemas de cambio (95\%) resultan más sencillos que los de igualación (91 \%), estos lo son más que los de combinación ( $88 \%$ ), que a su vez lo son más que los de comparación (81 \%) (Carpenter y otros, 1981). Y lo mismo puede decirse en general con respecto a niños preescolares o de segundo y tercero de EGB (Riley, 1981). No obstante, hay que indicar que el lugar que ocupa la incógnita constituye un factor aun más significativo que la estructura semántica del mismo problema, de modo que si el dato desconocido es la suma (todo), y no alguno de los sumandos (partes) entonces la resolución del problema resulta más sencilla (Bermejo y Rodríguez, 1986; De Corte y otros, 1985). En cuanto a la relación entre las estrategias empleadas por los niños y la estructura semántica de los problemas, Carpenter y Moser (1982) y Carpenter y otros (1981), han mostrado claramente este nexo en un estudio longitudinal, encontrando un conjunto de estrategias que, para abreviar, no recogemos aquí. Pero estas estrategias dependen también tanto de la edad de los niños (Riley, 1981; Riley y otros, 1983) como de la secuencia de los datos conocidos en el problema, tal como afirman De Corte y otros (1985): «la estrategia utilizada por los niños para resolver problemas elementales de suma y resta, no sólo depende de la estructura semántica de la tarea, sino también de la secuencia de elementos dados en el texto del problemav. (pp. 461-462). Igualmente, se ha encontrado que el tipo de error cometido por los niños depende tanto de la edad de los sujetos, como de la estructura semántica de los problemas planteados (ver, por ejemplo, De Corte y otros, 1985; Kiley, 1981). En consecuencia, la correcta resolución de las pruebas aditivas verbales parece depender de diferentes factores, sea de la edad de los niños, sea de la estructura semántica de los problemas, sea del lugar que ocupa la incógnita en la proposición de los mismos, sea de la secuencia que presentan los datos o conjuntos del problema, sea, en fin, del grado de explicitud que muestra el mismo texto verbal de la prueba.

Las investigaciones actuales se interesan preferentemente por los procesos cognitivos que conducen a la resolución de un problema, analizando a veces las categorías de errores producidas por los niños (De Corte y otros, 1985), y otras estudiando detalladamente cómo se llega a construir la representación mental del problema a partir del texto verbal del mismo. Desde esta óptica se insiste, con acierto a nuestro juicio, en que la resolución de problemas matemáticos verbales supone algo más que el mero conocimiento de operaciones (sumar, restar) y la habilidad para aplicarlas en situaciones concretas. La comprensión de los problemas verbales planteados presenta grandes semejanzas con la comprensión lingüística o de textos, de modo que la fase de ejecución del problema viene precedida de la fase de representación, que incluye, entre otras cosas, el procesamiento de la información contenida en el texto y una primera representación global del problema (De Corte y Verschaffel, 1985; Kintsch y Greeno, 1985). Asimismo, al igual que uno de los autores (Bermejo, 1985) puso de relieve en otro lugar el interés del factor lingüístico en la adquisición de conceptos lógicos, aquí también conviene enfatizar la relevancia de dicha dimensión. De hecho, las líneas principales seguidas en la investigación actual se ocupan directa o indirectamente de este aspecto, bien al estudiar la estructura semántica de los problemas (Carpenter y Moser, 1982; Vergnaud, 1982; Riley y otros, 1983); bien al analizar rasgos estructurales generales, como la complejidad gramatical del texto, por ejemplo (Carpenter y otros, 1981; Hudson, 1980; Jerman, 1971, 1973, 1974; Lindval e Ibarra, 1980; Rosenthal y Resnick,. 1974; Steffe y Johnson, 1971); bien al examinar los procesos mismos de representación (De Corte y otros, 1985; Kintsch y Greeno, 1985; Riley y otros, 1983); bien al estu- 
diar las estrategias utilizadas en la resolución de los problemas planteados (Carpenter y Moser, 1982; Fuson, 1982; Groen y Parkman, 1972).

Sin embargo, conviene señalar que si bien la comprensión de la estructura proposicional, así como los procesos de comprensión, son independientes de la temática concreta estudiada, no obstante, la representación de la situación o del problema requiere la incidencia de los conocimientos específicos del niño sobre la tarea, en orden a estructurar finalísticamente la información contenida en el texto. En este sentido, algunos modelos (Briars y Larkin, en prensa; De Corte y Verschaffel, 1985; Kintsch y Greeno, 1985) centran su análisis en el modo en que se lleva a cabo la representación del problema a partir del texto, suponiendo la presencia de una serie de procesos semánticos e inferenciales; mientras que otros (Greeno y otros, 1984; Riley y otros, 1983) especifican sobre todo los diferentes procesos que conducen a la solución del problema, o a posibles errores, a partir de la representación conceptual del mismo. En consecuencia, la estructura del problema planteado o la formulación verbal que se adopte para plantear dicho problema puede influir en el tipo de características de la representación global que se haga del mismo el sujeto. Pero una vez construida esta primera representación, peculiar a cada categoría de problemas, ise pasa a una representación conceptual para todos los problemas aditivos o, más bien, cada tipo de problema postula su propia representación conceprual específica? En otras palabras, jexiste un solo esquema general para representar cualquier problema aditivo o, por el contrario, cada problema o categoría de problemas requiere la construcción de un esquema específico?

Nuestro trabajo, teniendo en cuenta la ausencia de estudios españoles en torno al tema y la importancia que se está dando a la estructura del texto mismo de la prueba, que puede diferir según las distintas lenguas, asume que la estructura semántica del problema de combinación, por ejemplo, resultará más fácil que un problema de igualación, a pesar de que ambos impliquen la realización de la misma operación aritmética (adición). Ello podría implicar que, al menos en un principio, no existiría un esquema general que sirva para solucionar cualquier tipo de problema aditivo, sino, más bien, cada tipo de tarea (de cambio, combinación, igualación, etc.) requeriría la construcción, al menos provisional, de un esquema o algoritmo particular dependiente de su estructura semántica. Ahora bien, desde el punto de vista evolutivo y desde una óptica de análisis estructural, parece lógico suponer el paso de estas representaciones diferentes a una representación única y más general basada en el esquema parte-todo, que fundamentaría en definitiva la resolución de cualquier problema aditivo o de resta (Bermejo y Lago, 1986; Bermejo y Rodríguez, 1986; Resnick, 1983). En esta óptica estudiamos el comportamiento de los niños ante dos tipos de problemas diferentes: unos de combinación (Ej.: uJuan tiene 4 caramelos, María tiene 6, ¿cuántos tienen entre los dos?») y otros de igualación (Ej.: \&Juan tiene 6 caramelos, María tiene dos, ¿cuántos debería añadir María para tener los mismos que Juan?»); analizando evolutivamente tanto las categorías de respuestas emitidas por los niños como las estrategias de solución empleadas por los mismos. Igualmente, se tiene en cuenta la relevancia que puede tener la presencia de ayudas (objetos) durante la resolución de las pruebas, en orden a facilitar la representación y comprensión de las mismas. Esperamos que este examen nos permita hacer algunas observaciones de talante instruccional referentes, por ejemplo, al orden aconsejable en que deberían enseñarse algunos tipos de problemas aditivos o sobre la explicitud y secuenciación que han de presentar estos problemas con respecto a la relación entre las partes (sumandos) y el todo (suma). 


\section{METODOLOGIA}

\section{Sujetos}

Cien niños, elegidos al azar y pertenecientes a segundo de preescolar y primero de EGB de tres colegios nacionales de Madrid, pasan las pruebas. Se forman cuatro grupos de 25 sujetos cada uno, de manera que los dos primeros se componen de niños de segundo de preescolar y de primero de EGB los otros dos. El primero lo forman 13 niños y 12 niñas con edades comprendidas entre 5,0 a 5,6 años $(\vec{X}=5,4)$; el segundo está constituido por 15 niños y 10 niñas con edades que oscilan entre $5 ; 6$ a $6 ; 0$ años $(\bar{X}=5,8)$; el tercero corresponde a niños de $6 ; 0$ a $6 ; 6$ años $(\bar{X}=6,4)$, de los que 13 son niños y 12 niñas y, por último, el cuarto lo componen 16 niños y 9 niñas con edades entre $6 ; 6$ y 7;0 años $(\overline{\mathrm{X}}=6,8)$.

\section{Material y procedimiento empírico}

El material consiste en caramelos y un muñeco de guiñol. El experimentador presenta el muñeco como si estuviera aprendiendo a sumar, pidiendo al niño que le enseñe cómo realiza las adiciones. A continuación, invitándole a jugar con los caramelos, se plantean tareas aditivas. Se proponen dos tipos de problemas, de los que cuatro son de combinación (Ej.: «Yo tomo 4 caramelos y tú 5, ¿cuántos caramelos tenemos entre los dos?») y cuatro de igualación (Ej.: «Yo tomo 6 caramelos y tu 2, ¿cuántos caramelos deberías añadir para tener los mismos que yo?»), guardándose siempre este orden. En ambos casos, las cantidades utilizadas y los resultados obtenidos no superan el 9 con los niños preescolares, ya que en este curso sólo llegan a contar hasta esta cifra. En cambio, con los niños de $1^{\circ}$ de EGB se rebasa la decena dos veces en los problemas de combinación. La realización de las pruebas es individual y se hace en horas lectivas, con una duración aproximada de 15 minutos para cada niño.

\section{ANALISIS Y DISCUSION DE RESULTADOS}

En cuanto a los problemas de combinación, encontramos en una primera lectura que un alto porcentaje de niños de todas las edades responden acertadamente en todos los problemas (ver tabla I), de modo que los niños del G.I. llegan a resolver correctamente el $87 \%$ de las pruebas planteadas, el $91 \%$ delos del G.II, G.III resuelve el $94 \%$ y, finalmente, el $97 \%$ los niños mayores. Además, la mayoría de los errores cometidos se producen en la fase de ejecución y no en la de representación; es decir, los niños eligen la operación adecuada, pero fracasan en la cabal realización de la misma. El elevado éxito de estos niños, muy superior al obtenido en los problemas de igualación, como veremos después, podría deberse a dos factores principales: a la presencia de ayudas (objetos) y al lugar ocupado por la incógnita. El primero de estos dos aspectos es un factor facilitador en la solución de problemas aritméticos sobre todo en el caso de los niños pequeños, tal como han apuntado numerosos autores (Bolduc, 1970; Carpenter y Moser, 1982; Le Blanc, 1971; Marshall, 1977; etc.). Esta incidencia se halla claramente presente en nuestro estudio, ya que los niños disponen de caramelos perfectamente observables y manipulables, facilitando así la representación del problema y sobre todo la acción de contar. Conviene señalar aquí un efecto negativo que puede tener la disponibilidad de ayudas, consistente eńn inducir a los niños mayores a utilizar estrategias más simples que 
las que de hecho son capaces de emplear (Bermejo y Rodríguez, próxima aparición; Carpenter y Moser, 1982).

\section{TABLA I}

Porcentaje de niños que resuelven correctamente las pruebas de combinación

\begin{tabular}{|c|c|c|c|c|}
\hline \multicolumn{5}{|c|}{ SUJETOS } \\
\hline $\begin{array}{c}\text { No PRUEBAS } \\
\text { SOWCIONADAS }\end{array}$ & G.I & G.II & G.III & G.IV \\
\hline 4 & 84 & 88 & 80 & 92 \\
\hline 3 & 4 & 4 & 16 & 4 \\
\hline 2 & 0 & 0 & 4 & 4 \\
\hline 1 & 0 & 0 & 0 & 0 \\
\hline 0 & 12 & 8 & 0 & 0 \\
\hline
\end{tabular}

En relación con el lugar de la incógnita, un amplio conjunto de estudios (De Corte y Verschaffel, 1981; Grouws, 1971; Hirstein, 1979; Rosenthal y Resnick, 1974; Riley y otros, 1983; etc.) ponen de manifiesto los diferentes grados de dificultad en la resolución de problemas en función de la situación de la incógnita dentro de la ecuación. Las pruebas, en las que la incógnita se localiza en alguno de los dos sumandos $(?+a=b ; a+?=b)$, son más difíciles que aquéllas en las que el dato desconocido es el resultado $(a+b=$ ?). Esta dificultad ha sido explicada de manera diferente, diciendo bien que se trata de una forma indirecta (De Corte y Verschaffel, 1981), bien que se aparta de la forma canónica (Carpenter y Moser, 1983), bien por la cantidad de transformaciones que realiza el niño (Rosenthal y Resnick, 1974). Nuestros problemas toman la forma canónica o forma directa $(a+b=$ ?) y por tanto resultan fáciles de solucionar. Pero, a nuestro juicio, esta facilitación se debería además y sobre todo a que la incóg. nita se refiere al todo (resultado) y no a las partes (sumandos).

FIGURA 1

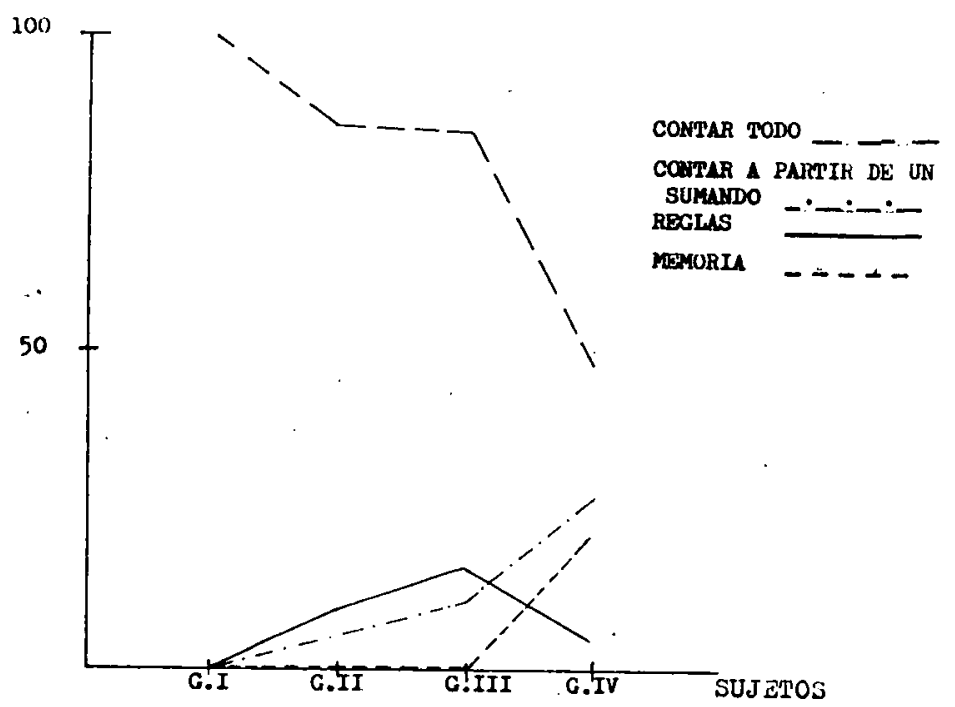


Con respecto a las estrategias utilizadas por los niños en la solución de los problemas de combinación (ver Figura 1), se observa en todos los grupos un elevado índice de niños que recurren al conteo de todos los elementos, haciéndolo de 3 formas distintas: contar con la mirada, contar señalando con el dedo y contar los dedos que representan los dos subconjuntos. Este monopolio, realmente importante, de la estrategia de contar podría deberse, al menos parcialmente y en los niños mayores (G.III y G.IV), a la disponibilidad de ayudas durante el tiempo de resolución de los problemas, tal como señalamos anteriormente y han sostenido otros autores (Carpenter y Moser, 1982; Fuson, 1982; Groen y Parkman, 1972; Siegler y Klahr, 1981). Pero estos mismos niños sobre todo utilizan otras estrategias más evolucionadas ( $\mathrm{Ej}$.: contar a partir de un sumando, reglas de descomposición y memorización) que exigen un nivel de abstracción mayor, si bien su uso alcanza porcentajes más bien bajos. Por otra parte, Riley y otros (1983) y De Corte y otros (1985) sugieren que el niño al oír la palabra acuántos» pondría en marcha un esquema de acción consistente en contar todos los caramelos como si el niño hubiera aprendido que la última palabra tras el conteo es la respuesta a la pregunta «cuántos». Si esto fuera así, la respuesta correcta no garantizaría ni la comprensión del problema, ni la representación mental del mismo en términos del esquema parte-todo. De hecho, la mayoría de los errores cometidos por los niños pequeños consisten en contar todo $(54,5 \%)$, cuando en realidad el problema postula otros procesos resolutivos, como veremos después, en los problemas de igualación. Vergnaud (1985) va más lejos al afirmar que la respuesta correcta mediante la estrategia de contar todo supone que el niño sabe contar, pero no que haya adquirido necesariamente la operación aditiva. En esta línea hay que interpretar, a nuestro juicio, los resultados aquí presentados. La respuesta correcta de los niños, sobre todo de los más jóvenes ( $\overline{\mathrm{X}}=5 ; 4$ años), no implicaría necesariamente la comprensión de la operación aditiva, ya que este tipo de problemas puede resolverse solamente mediante el conteo de todos los elementos. Ahora bien, cuando el niño cuenta todos los elementos, ¿lo hace porque ha entendido que la solución del problema supone la unión de las dos partes (sumandos) en un todo (suma o resultado)? Este interrogante, que requiere una nueva investigación, no puede encontrar la respuesta cabal en los resultados obtenidos aquí, si bien se ilustra al observar que el tipo de error más frecuente de los niños pequeños en los problemas de igualación consiste en contar todo, es decir, las dos cantidades propuestas en la prueba.

TABLA II

Porcentaje de niños que resuelven correctamente las pruebas de igualación

\begin{tabular}{|c|c|c|c|c|}
\hline \multicolumn{5}{|c|}{ SUJETOS } \\
\hline $\begin{array}{c}\text {N}^{0} \text { PRUEBAS } \\
\text { SOLUCIONADAS }\end{array}$ & G.I & G.II & G.III & G.IV \\
\hline 4 & 52 & 52 & 76 & 76 \\
\hline 3 & 0 & 12 & 12 & 12 \\
\hline 2 & 0 & 4 & 4 & 8 \\
\hline 1 & 4 & 4 & 0 & 4 \\
\hline 0 & 44 & 28 & 8 & 0 \\
\hline
\end{tabular}

En cuanto a los problemas de igualación (ver tabla II), sólo el $52 \%$ de los niños preescolares son capaces de solucionar correctamente todos los problemas, 
de manera que el G.I llega a emitir la respuesta exacta en el $53 \%$ de las tareas planteadas, el G.II obtiene un $64 \%$, un $87 \%$ los niños del G.III y, finalmente, los mayores responden acertadamente en el $90 \%$ de las pruebas propuestas. A la vista de estos resultados, es obvio que no todos los niños que resuelven los problemas de combinación son igualmente capaces de solucionar los de igualación. Al contrario, hay niños que resuelven bien los problemas de combinación y que, sin embargo, lo hacen erróneamente en los de igualación. Así, nueve niños pertenecientes al G.I, cuatro del G.II y dos del III, se encuentran en esta situación. En cambio, sólo un niño del G.II resuelve correctamente todos los problemas de igualación y ninguno de combinación. Por otra parte, la mayoría de los errores cometidos por los niños pequeños (G.I: $91,66 \%$; G.II: $61,5 \%$ ) se producen en la fase de representación de la información o fase de «pensars en palabras de De Corte $(1981,1985)$, presentando tres categorias principales de errores: contar todo, repetir una de las cantidades propuestas en la formulación del problema e inventar la respuesta. En estos casos, los niños preescolares parecen incapaces de construir la representación mental adecuada de la tarea encomendada, buscando entonces la solución, bien contando las dos cantidades propuestas en la prueba (contar todo), como si se tratase de un problema de combinación (42,1\%), bien dando la cantidad mayor que en la formulación verbal de la tarea corresponde al experimentador $(42,1 \%)$, bien inventando la respuesta. En cambio, apenas manifiestan esta dificultad los niños de $1^{\circ}$ de EGB, ya que sólo cuatro de ellos fracasan en este propósito.

Figura 2

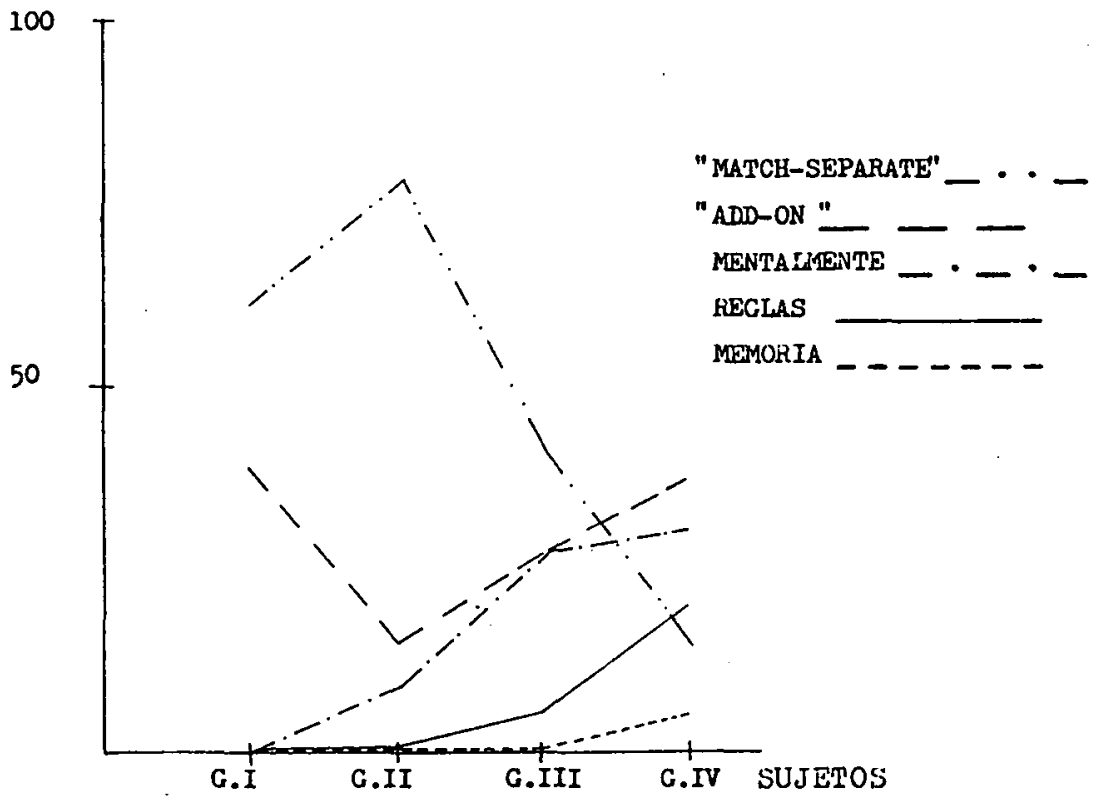

Estrategias de solución utilizadas en las pruebas de igualación

Las estrategias son, al menos en parte, función de la edad, tal como puede constatarse gráficamente en la Figura 2. El G.I utiliza exclusivamente el «match-separates y add-ons, en la terminología propuesta por Carpenter y Moser (1982). La estrategia \&match-separate consiste en formar un subconjunto en el conjunto más grande, en correspondencia con los miembros del conjunto más 
pequeño, contando después los elementos sobrantes; mientras que en la estrategia «add-on» el niño añade objetos al conjunto más pequeño hasta obtener el mismo cardinal que posee el conjunto mayor, contando después los elementos añadidos. En el G.II se acentúa más la utilización del ematch-separate» y disminuye el «add-on», iniciándose, al mismo tiempo, la manifestación de otras estrategias mentales que aún son incapaces de justificar. El G.III introduce una nueva estrategia, la aplicación de reglas, que consiste en realizar composiciones y descomposiciones de los números, y que supone un logro importante en este desarrollo conceptual, ya que implicaría la comprensión de que el todo puede dividirse o componerse indistintamente dentro del sistema sin alterarse el resultado final. Sin embargo, el porcentaje de sujetos que utiliza esta estrategia es todavía bajo. El G.IV emplea sobre todo el sadd-on» y la realización mental (Ej.: «Me imagino que te he cogido 2 y te quedan 4 , dice el niño ante el problema: «Yo tengo 6 caramelos, tú 2. ¿Cuántos deberías añadir para tener los mismos que yo?), siendo muy reducido el uso del amatch-separates. Es importante reseñar que estos niños son los que más emplean la estrategia consistente en la aplicación de reglas, iniciando también las estrategias memorísticas.

\section{CONCLUSIONES E IMPLICACIONES EDUCATIVAS}

El análisis de los datos recogidos en este trabajo permite discernir un incremento general con la edad en el porcentaje de niños que resuelven correctamente tanto los problemas de combinación como los de igualación (G.I: $48 \%$, G.II: $40 \%$, G.III: 68 \%, G.IV: $72 \%$ ). Pero al mismo tiempo resulta no menos manifiesto que estos porcentajes son netamente inferiores a los alcanzados sobre todo en las pruebas de combinación. Por tanto, puede colegirse, al menos provisionalmente, que la resolución correcta de un tipo de problemas no garantiza sin más una buena solución en otros similares. Este hecho podría imputarse con De Corte y Verschaffel (1981) a que en la enseñanza de las matemáticas se incide frecuentemente en el aprendizaje de métodos específicos y aislados, que sólo sirven para asegurar la cabal realización de determinado tipo de tareas, fallando sin embargo en aquellos otros problemas que, si bien requieren la misma competencia cognitiva, no son familiares a los niños. Otra posible interpretación que podría seguirse de la posición defendida por Vergnaud (1985), consiste en sostener que la respuesta exacta emitida en los problemas de combinación mediante la estrategia de contar todo mostraría obviamente que el niño sabe contar, pero no supondría necesariamente la comprensión de la operación aditiva. En cambio la resolución correcta de las pruebas de igualación implicaría con mayor probabilidad esa competencia.

En consecuencia, podemos concluir al menos dos cosas: por una parte, que desde el punto de vista empírico o factual hay problemas aditivos que resultan más fáciles que otros, debido fundamentalmente, como hemos visto más arriba, a su estructura semántica y al lugar que ocupa la incógnita o el dato desconocido en la ecuación $a+b=c$. Y esto queda suficientemente probado tanto por los resultados obtenidos en este trabajo como por los propuestos por la gran mayoría de los especialistas. Sería, pues, aconsejable que se tuviera en cuenta en la práctica educativa o instruccional el grado de dificultad que presentan los distintos problemas aditivos que se proponen a los niños para la adquisición de esta operación. Pero, por otra parte, habría que evitar los programas que tienden a formar expertos en algún tipo específico de problema, siendo estos niños incapaces de resolver tareas similares o de dificultad teóricamente parecida. $\mathrm{Pa}$ ra sortear esta inconveniente situación, pensamos que puede ser de gran utilidad el formular de manera explícita la relación partes-todo (sumandos-suma) 
en el texto de los problemas empleados, facilitando en cada caso concreto la comprensión de este esquema que subyace, a nuestro juicio, en todos los problemas aditivos. En este sentido, conviene que quede suficientemente explicitado en la verbalización de los problemas que los dos subconjuntos (sumandos) constituyen las partes de un todo (conjunto o suma). Uña vez que los niños logren identificar en las tareas aditivas las partes y el todo y comprendan las relaciones que los unen, gozarán entonces de un instrumento cognitivo de gran eficiencia para resolver las diferentes categorías de pruebas aditivas.

Por otra parte, la disponibilidad de ayudas (objetos) para resolver las tareas planteadas parece facilitar la representación y comprensión de los problemas concretos propuestos; sobre todo en los sujetos que se hallan en un momento de transición, es decir, que están adquiriendo esta operación. En este sentido, sería aconsejable la presencia de ayudas (objetos, dedos, etc.) para el aprendizaje de éstos conceptos. Sin embargo, conviene tener presente el efecto negativo que puede producir esta disponibilidad, al inducir el uso de estrategias más simples en niños mayores que pueden emplear otras más evolucionadas (Carpenter y Moser, 1982). Pero además, la presencia de objetos podría condicionar la elección de la misma operación utilizada, ya que ante un problema de igualación, por ejemplo, el niño tiende en general a resolverlo mediante el empleo de una estrategia aditiva si tiene a su disposición objetos; mientras que el mismo niño lo haría probablemente restando ante la ausencia de tales ayudas. En consecuencia, resulta ventajoso en general el uso de objetos, dedos, dibujos, etc., para la enseñanza de la operación aditiva; pero hay que ser conscientes de los efectos contraproducentes que en ciertas condiciones podrían causar.

\section{Resumen}

Se analizan los diferentes factores que pueden influir en la soluciōn de problemas verbales aditivos, insistiēndose sobre todo en la incidencia de la estructura semántica de los mismos y en su expresión verbal. Dos grupos de niños de segundo de preescolar y otros de primero de EGB pasan pruebas aditivas de combinación e igualación. Los resultados confirman que la dificultad de las tareas depende, entre otras variabes, de la estructura semántica de las mismas, del lugar que ocupa la incógnita o dato desconocido y de la disponibilidad de ayudas en la resolución de los problemas. A demás, las estrategias utilizadas por los niños son función de la edad de los mismos y del tipo de problema planteado. Se señalan también algunas de las implicaciones educativas que se desprende de este trabajo.

\section{Summary}

The different factors which can contribute to solving additional verbal problems by emphasizing their semantic structure's influence and their verbal formulate. Were analyzed two groups of children from the last year of kinder. garden and two others from the first year of primary school, were applied combine and equalize addition tests. Results confirmed that difficulty of tasks mainly depends upon their semantic structure, the place which the unk. nown occupies, and the available belp during the time the problem was being solved. On the other hand, the strategies the children use are a function of their age, and the category of the given problem. Some of the educative implications which arise from this work are also indicated. 
Anderson, J.; Greeno, J.; Kune, P. y Neves, D. (1981). Acquisition of problem-solving skill. En J. R. Anderson (Ed.), Cognitive skills and their acquisition. Hillsdale, N. J.: Lawrence Erlbaum Associates.

BERMEjo, V. (1985). Estudio evolutivo de las conductas de clasificación en el niño. Aspectos lingüísticos y perceptivos. Infancia y Aprendizaje, 31-32, 211-227.

BERMEJO. V. y LAGO, M. O. (1986). La adquisición de la adición. Estrategias infantiles en función de la naturaleza de los sumandos. Segundas Jomadas Internacionales de Psicología y Educación, Madrid, Junio.

BERMEjo. V. y RoDrfGUEZ , P. (1986). El esquema parte-todo en la conservación y la adición. Segundas Jomadas Intemacionales de Psicología y Educaciōn, Madrid, Junio.

Bermejo. V. y Rodríguez, P.: Fundamentos cognitivos de la adición. Psiquis, (en prensa).

BISHOP, A. (1983). Space and Geometry. En R. Lesh y M. Landau (Eds.), Acquisition of mathematics concepts and processes. N. York: Academic Press.

BRIARS, D. y LARKIN, J.: An integrated model of skill in solving elementary word problems. Cognition and Ins. truction (en prensa).

CARPENTER, T.; HIEBERT. J. y MOSER, J. (1981). The effect of problem structure on firsi arader's initial solution processes for simple addition and subtraction problems. Joumal for Research in Mathematics Education, 12(1), $27-39$.

CARPENTER, T. y MOSER. J. (1982). The development of adition and subraction problem-solving skills. En T. Carpenter, J. Moser y T. Romberg (Eds.), Addition and subtracion: A cognitive perspective. (pp. 9-24). Hillsdale, N. J.: Erlbaum.

CARPENTER, T. y MOSER. J. (1983). The adquisition of addition an subtraction concepts. En R. Lesh y M. Landau (Eds.), Acquisition of mathematics concepts and processes (pp. 7-44). N. York: Academic Press.

De CorTe, E. y Verschaffei, L. (1981). Children's solution processs in elementary arithmetic problems: Analysis and improvement. $73,775-779$.

DE CORTE, E.; VERSCHAFFEL, L. y DE WIN, L. (1985). Influence of rewording verbal problem on children's problem representations and solutions. Journal of Educational Psycbology, 77, 460-470.

DUNCKer, K. (1945). On problem solving. Psychological Monographs, 1945, 58 (270), 1-112.

FUSON, K. (1977). The counting-on solution procedure: Analysis and empirical results. En T. Carpenter, J. Moser y T. Romberg (Eds.), Addition and subtraction: $A$ cognitive perspective. Hillsdale N. J.: Lawrence Erlbaum Associates.

GrEENO, J. (1978). A study of problem solving. En R. Glaser (Ed.), Advances in instructional psychology (Vol. 1). Hillsdale, N. J.: Lawrence Erlbaum Associates.

Greeno, J.; RILEY, M. y Gelman, R. (1978). Young children's counting and understanding. Comunicación presentada al encuentro anual de la Psychonomic Society, San Antonio.

Groen, G. y PARKMan, J. (1985). A chronometric analysis of simple addition. Psycbological Review, 92(1), $109-129$.

Grows, D. A. (1972). Differential performance of third-grade children in solving open sentences of four types. (Defensa de tesis, University of Wisconsin, 1971). Dissertation Abstracts Intermational, 32, $3860 \mathrm{~A}$.

HIRSTEIN. J. (1979). Children's counting in addition, subtraction, and numeration contexts (Defensa de tesis, University of Georgia, 1978). Dissertation Abstraction Intermational, 39, 7203 A.

Hudson, T. (1980). Young children's difficulty with How many more.... than ...... are there? questions (Defensa de tesis, Indiana University, 1980). Dissertation Abstract International. 41, (01).

Hudson, T. (1983). Correspondences and numerical differences between disjoint sets. Child Development, 54, 84-90.

JERMAN, M. (1971). Instruction in problem solving and analysis of structural variables that contribute to problemsolving difficulty (Tech. Rep. No 1807. Stanford: Institute for Mathematical Studies in the Social Sciences.

Jerman, M. (1973). Problem length as a structural variable in verbal arithmetic problem. Educational Studies in Mathematics, 5, 109-123.

JERMAN, M. (1974). Linguistic and computational variables in problem solving in elementary mathematics. Educational Studies in Matbematics, 5, 317-362.

KINISCH, W. y GREeno, J. (1985). Understanding and solving word arithmetic problems. Psychological Review, 92(1), 109-129.

KOHLER, W. (1927). The mentality of apes. New York: Harcourt Brace.

LARKIN, J. (1985). Understanding problems representation and skill in physics. En S. Chipman, J. Segal y R. Glases (Eds.), Thinking and learning skills (Vol. 2). Hillsdale, N. Jersey: Lawrence Erlbaum Associates.

LINDVAL, C. e IBARRA, C. (1980). Incorrect procedures used by primary grade pupils in solving open addition and subtraction sentences. Journal for Research in Mathematics Education, 11, 50-62.

Maitzman, I. (1955). Thinking: From a behavioristic point of view. Psychological Review, 62, 275-286.

McDERMOTT, J. y LARKIN, J. (1978). Representing textbook physics problems. En Proceedings of the Second National Conference, Canadian Society for Computational Studies of Intelligence. Toronto, Canada: Universidad de Toronto.

NovaK, G. (1976). Computer understanding of physics problems staked in natural language. American Journat of Computational Linguistics, (Microficha 53).

RESNICK. L. (1982). Syntax and semantics in learning to subtract. En T. Carpenter, J. Moser y T. Romberg (Eds.), Addition and subtraction: A cognitive perspective. Hillsdale, N. J.: Erlbaum.

RILEY, M. (1981). Conceptual and procedural knowledge in development. Tesis de licenciatura inédita, University of Pittsburg.

RILEY, M.; GREENO, J. y HeILER, J. (1983). Development of children's problem-solving ability in arithemetic. En H. Ginsburg (Ed.), The development of mathematical thinkind. N. York: Academic Press.

RosenthaL., D. y RESNICK, L. (1974). Children's solution processes in arithmetic word problems. Joumal of Educational Psychology. 66, 812-825.

SIEGLER, R. y KLAHR, D. (1981). When do children learn? The relationship between existing knowledge and the 
acquisition of new knowledge. En R. Glaser (Ed.), Advances in instructional psychology (Vol. 2). Hillsdale, N. J.: Lawrence Erlbaum Associates.

STEFF, L. y JOHNSON, D. (1971). Problem-solving performance of first-grade children. Joumal for Research in Matbesmatics Education, 2, 50-64.

UNDERWWOOD, B. y RICHARDSON, J. (1956). Some verbal materials for the study of concept formation. Psychological Bulletin, 53, 84.95.

VERGNAUD, G. (1982). A classifiction of cognitive tasks and operations of thought involved in addition and subtraction problems. En T. Carpenter, J. Moser y T. Romberg (Eds.), Addition and substracion: A cognitive perspective (pp. 39-59). Hillsdale, N. J.: Erlbaum.

VERGNAUD, G. (1985). Concepts et schémes dans une théorie opératoire de la représentation. Psychologie Franfazse, $30,245 \cdot 252$.

WOLIERS. M. (1983). The part-whole schema and arithmetic problems. Educational Studies in Mathematics, 2, 127-138.

WEAVER, J. (1971). Some factors associated with pupils performance levels on simple open addition and subtraction sentences. The arithmetic Teacher, 18, 513-519.

WERTHEIMER, M. (1959). Productive thinking. N. York: Harper \& Row, (Edic. revisada). 